\title{
Experimental Demonstration of Flex-Grid udWDM with 6.25GHz Full-Duplex Frequency Slots for Metro/Access \& Data Centers
}

\author{
Jose A. Altabas ${ }^{(1)}$, David. Izquierdo(l)(2), Felix Sotelo ${ }^{(1)}$, \\ Ignacio Garces ${ }^{(1)}$ \\ (I) Aragon Institute of Engineering Research (I3A) \\ Universidad de Zaragoza \\ Zaragoza, Spain \\ \{jaltabas, d.izquierdo,fsotelo,ngarces\}@unizar.es
}

\author{
Jose A. Lazaro ${ }^{(3)}$, Adolfo Lerin ${ }^{(3)}$, Salvatore Spadaro ${ }^{(3)}$, \\ Gabriel Junyent ${ }^{(3)}$ \\ (2) Centro Universitario de la Defensa \\ Academia General Militar \\ Zaragoza, Spain \\ (3) Universitat Politècnica de Catalunya (UPC) \\ \{jose.lazaro,adolfo.lerin,spadaro,junyent\}@tsc.upc.edu \\ Barcelona, Spain
}

\begin{abstract}
Flex-Grid $6.25 \mathrm{GHz}$ full-duplex frequency slots are proposed for Metro/Access \& Data-Center Networks allocating: 1Gbps Nyquist-DPSK over MZM for OLT/DC-ONU downlinks and OLT/DC up-downlinks; 1Gbps NRZ-DPSK over directly modulated DFB for ONU-OLT/DC uplinks; with $50 \mathrm{~km}$ single fiber transmission.
\end{abstract}

Keywords-Flex-Grid, udWDM, Nyquist pulse shaping, heterodyne detection

\section{INTRODUCTION}

The ICT eco-system has quickly evolved during the last years. Growing cloud and multimedia streaming services are creating new communication frameworks, requiring flexible architectural changes in order to enable scalability while supporting a high level of dynamic connectivity and accessing to Data Centers (DC), either directly connected to Metro networks, as depicted in Fig. 1, or at higher level regional and core networks. While Core maintains as multi-layer packet over optical networks, Metro networks are merging with

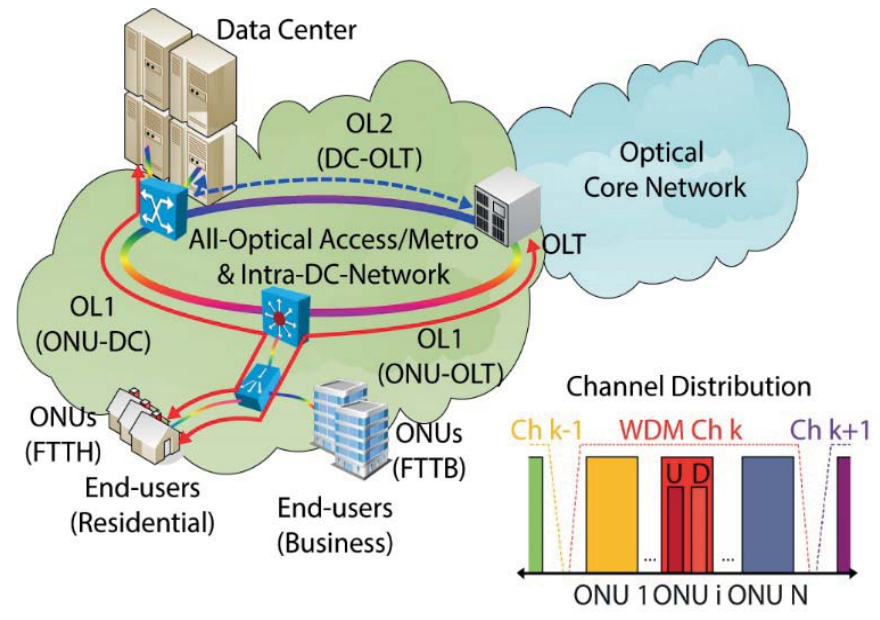

Fig. 1. All-optical Access/Metro \& DC Network scenario. Inlet: proposed Flex-Grid 6.25GHz full-duplex FS
Access networks, and evolving towards all-optical solutions [1-2]. Moreover, the increasing growth of traffic demand is pushing an efficient use of optical network resources. Flexible Optical Networks and bandwidth optimization, e.g. by Nyquist shapers, are developing as a technological solution [3]. Flexible digital transmitters and receivers are experimentally investigated in this paper, both: for ONU (Optical Network Unit) -to-DC/OLT (Optical Line Terminal) (OL1 in Fig. 1) (based on cost-effective laser TX) and DC-to-OLT (OL2 on Fig. 1), higher performance, providing compact spectrum use thanks to achieving full-duplex links over $6.25 \mathrm{GHz}$ udWDM channels, proving a Flex-Grid.

The full-duplex 1Gbps optical links proposed in this paper use $6.25 \mathrm{GHz}$ Frequency Slots (FS) allocation both: downlink (from OLT/DC to ONU) and uplink (from ONU to OLT/DC). This slot is obtained dividing an ITU $100 \mathrm{GHz}(200 \mathrm{GHz})$ WDM channel into 16 (32) $6.25 \mathrm{GHz} F S$, allowing 32Gbps (64Gbps) multicarrier channels.

\section{EXPERIMENTAL SETUP}

The experimental setup of the two full-duplex links proposed for the all-optical network is shown in Fig. 2. The first optical link (OL1) connects the OLT or DC with several ONUs and the second link (OL2) connects the OLT with DC.

OLT and DC Transmitters are based on external cavity Tunable Laser Sources (TLS), with $100 \mathrm{kHz}$ linewidth, which are phase-modulated using Mach-Zehnder Modulators (MZM). ONU transmitters consist of direct-phase-modulated Distributed Feedback Lasers (DFB) JDS Uniphase CQF915/1839, exhibiting relative wide linewidth, $>10 \mathrm{MHz}$. DFBs are equalized for direct-modulation of the laser phase as explained in [4], and their emission wavelengths are thermally tuned to achieve the channel spacing and flexible grid requirements inside the WDM channel.

1Gbps DPSK data-streams are encoded at the Digital Transmitters (DTX). The OLT and DC transmitted symbols are 


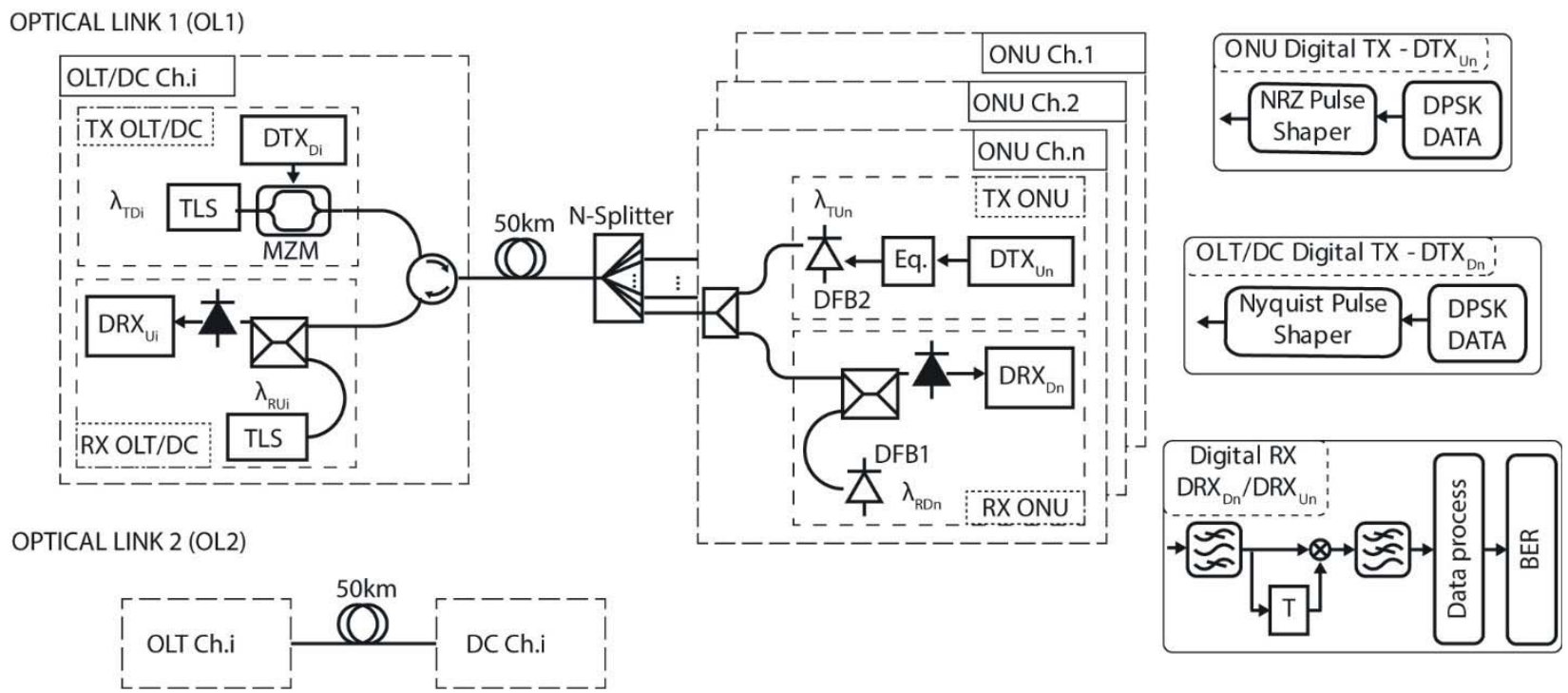

Fig. 2: Experimental setup of the two optical links.

generated by a Nyquist Pulse Shaper filter (12-symbols filter length and roll-off factor of 0 ) inside DTX $\mathrm{Di}_{\mathrm{Di}}$. The ONU transmitted symbols are Non Return to Zero (NRZ) formatted in $\mathrm{DTX}_{\mathrm{Ui}}$. All the signals are digitally generated at $4 \mathrm{GSa} / \mathrm{s}$ using an Arbitrary Waveform Generator (Keysight 81180B).

The operating point of the MZMs and the DFBs equalization have been adjusted to obtain a phase shift of $180^{\circ}$ between symbols using an High-Resolution Complex Spectrum Analyzer (Aragon Photonics BOSA Complex Spectrum Analyzer).

The receivers are based on a single photodetector heterodyne detection (DSC-R402PIN). The Local Oscillators (LO) used in the OLT/DC and ONU receivers are also an external cavity TLS and a DFB, respectively. The wavelengths of these LOs are tuned $2 \mathrm{GHz}$ away from the transmitter wavelength, being $\lambda_{\mathrm{RDi}}=\lambda_{\mathrm{TDi}}+\Delta \lambda(2 \mathrm{GHz})$ at the ONU and $\lambda_{\mathrm{RUi}}=\lambda_{\mathrm{TUi}}-\Delta \lambda(2 \mathrm{GHz})$ at the OLT/DC. Although the coherent detection is highly dependent of the polarization, our system is easily upgradeable to a polarization insensible heterodyne receiver lithe the proposed in [6].

The received signal, after the heterodyne detector, has been optically down-converted to an Intermediate Frequency (IF) equal to $2 \mathrm{GHz}$ (exactly the frequency shift between the LO and the central wavelength of transmission). The IF signal passes through a DC-Block and it is digitalized with Tektronik 40GSa/s Digital Oscilloscope (DPO 7254). The first step in the digital processing is the bandpass filtering of the digitalized signal with a FIR filter of $0.5-3.5 \mathrm{GHz}$ bandwidth in order to eliminate the adjacent channels and reduce the noise. Then, to demodulate the DPSK format, the signal is multiplied with itself delayed one symbol, Fig. 2, and lowpass filtered with a FIR filter of $1.5 \mathrm{GHz}$ bandwidth. Finally, the BER is calculated comparing the detected data-stream with the original one.

\section{RESULTS}

The performance of both links, Nyquist-DPSK modulated MZMs and NRZ-DPSK directly-modulated DFBs, has been analyzed to proof the feasibility of the proposed flexible udWDM full-duplex optical links.

The 1Gbps Nyquist-DPSK transmission used in the downlink from OLT to ONU and in OLT-DC links, achieves the FEC limit of $\mathrm{BER}=2.2 \cdot 10^{-3}$ [5] with a received power of $-47 \mathrm{dBm}$ in back-to-back (btb) connection, as is shown at Fig. 3. The transmission through a $50 \mathrm{Km}$ optical fiber only causes a power penalty of $1 \mathrm{~dB}$. On the other hand, the 1Gbps NRZDPSK transmission used for the uplink (from ONU to OLT) requires, in btb connection, at least $-43.5 \mathrm{dBm}$ of received power to have a BER below of the same FEC limit. In this case, the power penalty of a $50 \mathrm{Km}$ fiber-long transmission is around $2 \mathrm{~dB}$.

The BER penalty when uplink and downlink of the same and different channels are active simultaneously has been studied to ensure the feasibility of the $6.25 \mathrm{GHz}$ Frequency Slot and to determine the frequencies distribution of the optical links inside it. This BER penalty, obtained as the BER degradation from an initial value of $10^{-3}$, is measured for several distances between the central frequencies of the

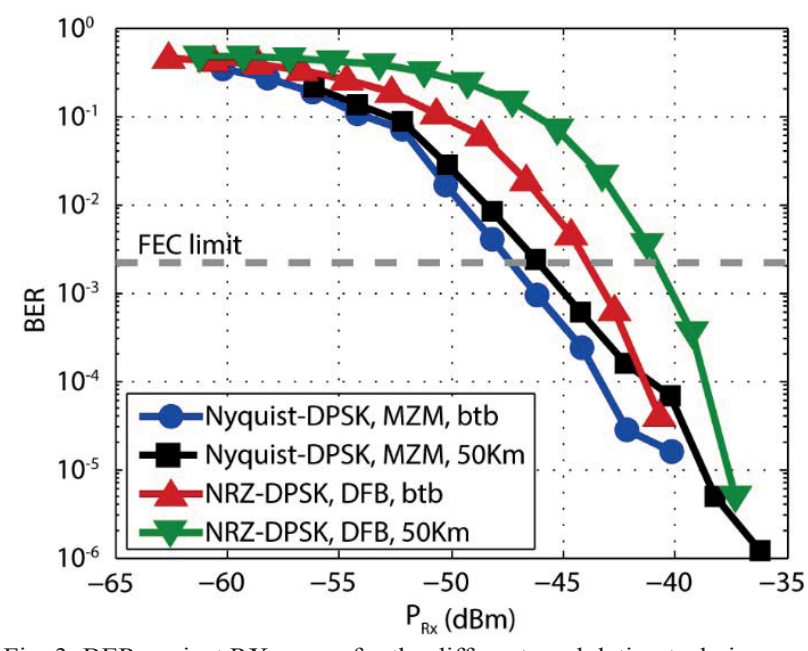

Fig. 3: BER against RX power for the different modulation techniques. 


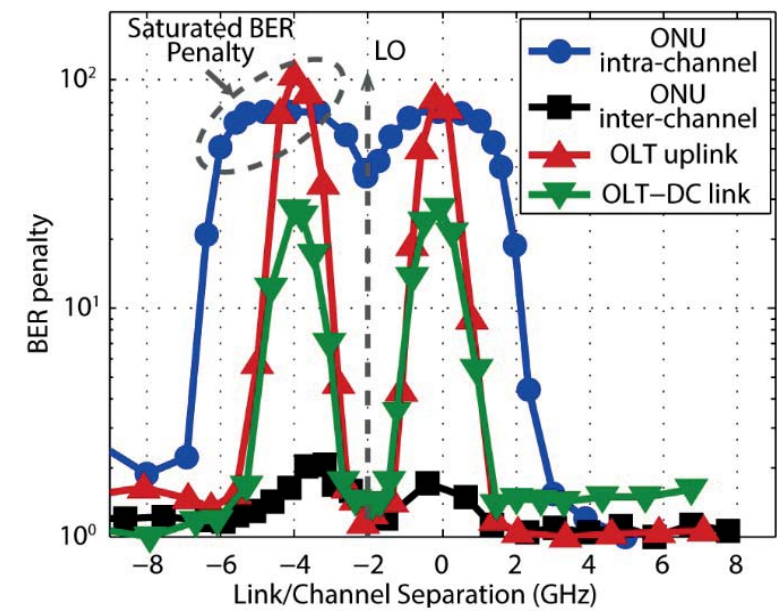

Fig. 4: Ber penalty for different links: ONU downlink, OLT uplink and OLTDC uplink and downlink.

interference link and the link under study.

In the first case, ONU intra-channel, the BER penalty in the ONU downlink is obtained when the same ONU is transmitting the uplink of the same channel (Ch.i). Fig. 4 shows the uplink central frequency $\left(\lambda_{\mathrm{TUi}}\right)$ cannot be located in the $-7 \mathrm{GHz}$ to $+3 \mathrm{GHz}$ frequency range from the center of the downlink $\left(\lambda_{\mathrm{DUi}}\right)$.

In ONU inter-channel case, the BER penalty of the ONU downlink (Ch.i) is obtained when the adjacent channel ONU is transmitting the uplink (Ch.i-1). The maximum BER penalty is smaller than in the intra-channel case due to the reduction of the backscattering power $(24 \mathrm{~dB}$ using a 1:16 splitter for the downlinks distribution). Also, a smaller banned frequencies range for the adjacent channel uplink is obtained, from $-5 \mathrm{GHz}$ to $1 \mathrm{GHz}$ from the center of the downlink frequency.

In OLT uplink case, the OLT is receiving the ONU uplink (Ch.i) and, simultaneously, the OLT is transmitting a downlink (Ch.i or Ch.i+1). The downlinks cannot be allocated in two frequency ranges: $-5 \mathrm{GHz}$ to $-2.75 \mathrm{GHz}$ and $-1.25 \mathrm{GHz}$ to $1 \mathrm{GHz}$ from the center of the uplink frequency. The important reduction of the BER penalty around the OLT LO frequency is due to the narrow spectral bandwidth of the Nyquist-DPSK transmission used in the downlink $(1 \mathrm{GHz})$.

In OLT-DC link case, the OLT is receiving the DC uplink while it is transmitting the downlink in the same channel to the DC. This downlink cannot be allocated at the same channel distances than in the OLT-uplink case.

The results of the BER penalty determine the channel allocation in order to obtain full-duplex links in the $6.25 \mathrm{GHz}$ FS as shown in Fig. 5, where three contiguous channels spectrum is plotted. The distance between the downlink and the uplink of the same channel in the OL1 (OLT/DC-ONU) must be $3.75 \mathrm{GHz}$. The ONU LO is $2 \mathrm{GHz}$ down of the downlink central frequency while the OLT LO is placed $2 \mathrm{GHz}$ up of the uplink center. Thus, the distance between the downlink of adjacent channel $(\mathrm{Ch} . \mathrm{i}+1)$ and the uplink of main channel (Ch.i) is $2.5 \mathrm{GHz}$. The same channel distribution can be used at the OL2 (OLT-DC link) without any BER penalty.

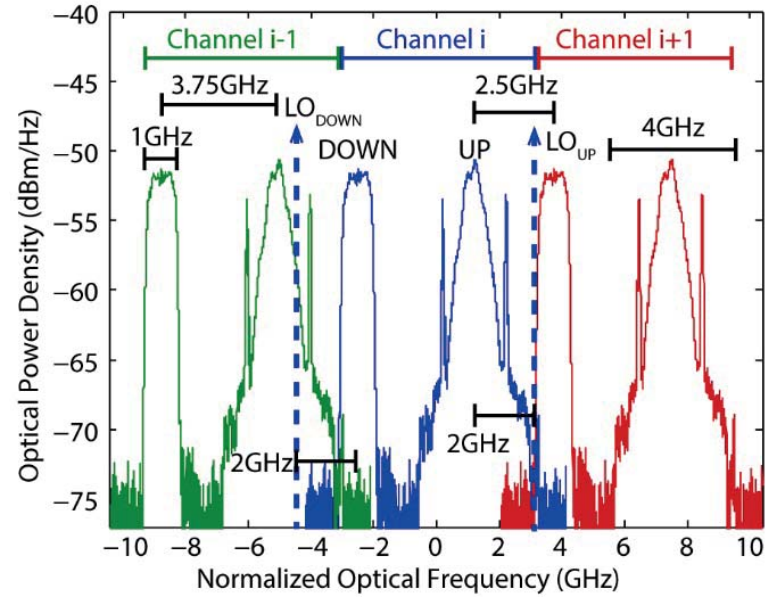

Fig. 5. Spectrum of three contigous OLT/DC-ONU channels. The central frequency corresponds to a $1560.3 \mathrm{~nm}$.

\section{CONCLUSION}

This paper shows the performance of a full-duplex optical link for a cost-effective udWDM in flexible Metro-Access Networks. The 1Gbps OLT downlinks and DC uplink are Nyquist-shaped DPSK modulation implemented using MZM with a sensitivity of $-47 \mathrm{dBm}$ (FEC limit) at ONU receiver. The 1Gbps ONU uplink uses a NRZ-DPSK directly-modulated DFB with a sensitivity of $-43.5 \mathrm{dBm}$ (FEC limit) at the OLT receiver. All the receivers are based on single photodiode heterodyne detection with a local oscillator (TLS in OLT/DC, and DFB in ONU).

The BER penalty analyzed in this paper shows that both of the links (uplink and downlink) can be allocated in a $6.25 \mathrm{GHz}$ FS. Thus, the full-duplex optical link has been experimentally demonstrated for flexible metro-access \& DC cost-effective udWDM networks.

\section{ACKNOWLEDGMENT}

This work was partially supported by the Diputación General de Aragón under grant T25, the Spanish MICINN projects muCORE (TEC2013-46917-C2-2-R) and SUNSET (TEC2014-59583-C2-1-R).

\section{REFERENCES}

[1] H. Song et al., "Long-reach optical access networks: A survey of research challenges, demonstrations, and bandwidth assignment mechanisms," IEEE Comm. Surv. \& Tut., Vol. 12, no. 1, p. 112, 2010.

[2] J.A. Lazaro et al., "Scalable extended reach PON," Proc. OFC, OThL2, Anaheim, 2008.

[3] J.A. Altabas et al., "Experimental Bandwidth Optimization for Flexible PON Using Nyquist Shaped PSK," Proc. CLEO/Europe-EQEC 2015, CI-2.4, Munich, 2015, accepted for publication.

[4] I.N. Cano et al., "Direct Phase Modulation DFBs for Cost-Effective ONU Transmitter in udWDM PONs," Photon. Technol. Lett., Vol. 26, no. 10 , p. 973 (2014).

[5] ITU-T Rec., G.975.1 (2004).

[6] I.N. Cano et al., "Simplified polarization diversity heterodyne receiver for $1.25 \mathrm{~Gb} / \mathrm{s}$ cost-effective udWDM-PON," Proc. OFC, W4G.2, San Francisco, 2014. 